\title{
$n$-FRAMES IN EUCLIDEAN $k$-SPACE
}

\author{
J. C. CANTRELL
}

1. Introduction. An $n$-frame $F_{n}$ is a union of $n$ arcs, $F_{n}=\bigcup_{i=1}^{n} A_{i}$, with a distinguished point $p$ such that, if $n=1, p$ is an end point of $A_{1}$, and if $n>1, p$ is an end point of each $A_{i}$ and $A_{i} \cap A_{j}=p, i \neq j$. We introduce the distinguished point in order to differentiate between a 1-frame and a 2-frame. A 1-frame is an arc with an end point distinguished and a 2 -frame is an arc with an interior point distinguished. This difference will keep certain logical difficulties from arising in the inductive proof of Theorem $1_{n}$.

In $E^{k}$ let $B_{i}$ be the arc in the $x_{1}, x_{2}$ plane, defined in polar coordinates by $r \leqq 1, \theta=\pi(1-1 / i)$. For $n$ a positive integer, the standard $n$-frame $G_{n}$ is defined by $G_{n}=\bigcup_{i=1}^{n} B_{i}$. An $n$-frame $F_{n}$ in $E^{k}$ is said to be tame if there is a homeomorphism of $E^{k}$ onto itself which carries $F_{n}$ onto $G_{n}$. Otherwise $F_{n}$ is said to be wild. For $n>1, F_{n}$ is said to be mildly wild if it is wild and $F_{n}-\left(A_{i}-p\right)$ is tame for $i=1,2, \cdots, n$. In [3] it was shown that for each $n>1$ there are mildly wild $n$-fra mes in $E^{3}$. Since there are wild arcs in $E^{k}$ for each $k>3$ [1], there will be wild $n$-frames for these dimensions. However, we will show that there are no mildly wild $n$-frames in $E^{k}$ for $k>3$. It then follows that, for $k>3$, the union of two tame arcs meeting only in a common end point is a tame arc. With a small amount of additional argument we will show that a wild arc (simple closed curve) in $E^{k}, k>3$, must fail to be locally flat at each point of some Cantor set. (If $S$ is an arc (simple closed curve) in $E^{k}$, we say that $S$ is locally flat at $p \in S$ if there is a neighborhood $U$ of $p$ and a homeomorphism $h$ which carries $U$ onto $E^{k}$ with $h(U \cap S)$ lying in the $x_{1}$-axis.)

Through the remainder of this paper we will assume that we are working in an euclidean space $E^{k}$ with $k>3$. We recall that for an arc or simple closed curve in $E^{k}$ to be tame it is sufficient that it be locally flat at each of its points. This result for simple closed curves is proved in [5]. The same technique of proof may be used to establish the corresponding result for arcs.

2. Basic lemmas. In [4] it was stated that the result contained in Lemma 2 below followed as a corollary to a theorem concerning manifolds with boundary $E^{k-1}$ and interior $E^{k}$. Since it seems that a more

Presented to the Society, August 29, 1963; received by the editors April 22, 1963. 
direct proof should be available to the reader, an alternate proof is included in this paper.

LEMma 1. Let $L$ be an arc in $E^{k}, p$ an end point of $L$, and $U$ a neighborhood of $L-p$. If $L$ is locally flat at each point of $L-p$, then there is a homeomorphism $f$ of $E^{k}$ onto itself such that $f$ is the identity outside $U$ and $f(L)$ is locally polyhedral at each point of $f(L-p)$.

Proof. Let $p_{0}$ be the end point of $L$, different from $p$, and let $p_{1}, p_{2}, \cdots$ be a sequence of points of $L$ converging to $p$ with $p_{0}<p_{1}$ $<p_{2}<\cdots$ relative to the order of $L$ from $p_{0}$ to $p$. For each integer $i$ let $\epsilon_{i}=1 / i$ and let $A_{i}$ be the closed subarc of $L$ from $p_{0}$ to $p_{i}$. Since $A_{2}$ is tame, we may select a closed $k$-cell neighborhood $U_{1}$ of $A_{1}$ with the properties: (1) $U_{1}$ is contained in the $\epsilon_{1}$-neighborhood of $A_{1}$ and in $U$, (2) $U_{1} \cap\left(L-A_{2}\right)=\square$, and (3) $U_{1}$ may be assigned a combinatorial triangulation in which $A_{1}$ is polyhedral. We then apply Homma's Theorem [5] to obtain a homeomorphism $f_{1}$ of $E^{k}$ onto itself such that $f_{1}$ is the identity outside $U_{1}$ and $f_{1}\left(A_{1}\right)$ is polyhedral in $E^{k}$.

Assume that for each integer $i>1$ certain homeomorphisms $f_{i-1}, \cdots, f_{1}$ of $E^{k}$ onto itself have been constructed so that $f_{i-1} \cdots$ $f_{2} f_{1}\left(A_{i-1}\right)$ is polyhedral in $E^{k}$. If $i=2$, let $U_{2}$ be a closed $k$-cell neighborhood of $\mathrm{Cl} f_{1}\left(A_{2}-A_{1}\right)$ with the properties: (1) $U_{2}$ is contained in the $\epsilon_{2}$-neighborhood of $f_{1}\left(A_{2}-A_{1}\right)$ and in $U$, (2) $U_{2} \cap f_{1}\left(L-A_{3}\right)=\square$, and (3) $U_{2}$ may be assigned a combinatorial triangulation in which $U_{2} \cap f_{1}\left(A_{2}\right)$ appears as a polyhedron. We then apply Theorem 2.1 of [5] to obtain a homeomorphism $f_{2}$ of $E^{k}$ onto itself such that $f_{2}$ is the identity outside $U_{2}$ and on $f_{1}\left(A_{1}\right)$, and $f_{2} f_{1}\left[\mathrm{Cl}\left(A_{2}-A_{1}\right)\right]$ is polyhedral in $E^{k}$. Note that at this point $f_{2} f_{1}\left(A_{2}\right)$ is polyhedral in $E^{k}$. If $i>2$, since $A_{i+1}$ is tame, we may select a closed $k$-cell neighborhood $U_{i}$ of $\mathrm{Cl} f_{i-1} \cdots f_{2} f_{1}\left(A_{i}-A_{i-1}\right)$ with the properties:

(1) $U_{i}$ is contained in the $\epsilon_{i}$ neighborhood of $f_{i-1} \cdots f_{2} f_{1}\left(A_{i}-A_{i-1}\right)$ and in $U$,

(2) $U_{i} \cap\left[f_{i-1} \cdots f_{2} f_{1}\left(L-A_{i+1}\right)\right]=\square$,

(3) $U_{i} \cap\left[U_{j=1}^{i-2} U_{j}\right]=\square$,

(4) $U_{i} \cap\left[U_{j=1}^{i-2} f_{i-1} \cdots f_{2} f_{1}\left(U_{j}\right)\right]=\square$, and

(5) $U_{i}$ may be assigned a combinatorial triangulation in which $U_{i} \cap f_{i-1} \cdots f_{2} f_{1}\left(A_{i}\right)$ is polyhedral. Again Theorem 2.1 of [5] is applied to obtain a homeomorphism $f_{i}$ of $E^{k}$ onto itself such that $f_{i}$ is the identity outside $U_{i}$ and on $f_{i-1} \cdots f_{2} f_{1}\left(A_{i-1}\right)$, and

$$
f_{i}\left[\mathrm{Cl} f_{i-1} \cdots f_{2} f_{1}\left(A_{i}-A_{i-1}\right)\right]
$$

is polyhedral in $E^{k}$. 
For each $x \in E^{k}$ we set $f(x)=\lim _{i \rightarrow \infty} f_{i} \cdots f_{2} f_{1}(x)$. Depending principally on the fact that if $x \in \bigcup_{j=1}^{\infty} U_{j}, f(x)=x$, and if $x \in U_{j}, f(x)$ $=f_{j+1} f_{j} f_{j-1}(x)$ one establishes that $f$ is a homeomorphism of $E^{k}$ onto itself, and $f(L)$ is locally polyhedral at each point different from $f(p)$.

Lemma 2. If $L$ is as in Lemma 1, then $L$ is tame.

Proof. Let $f$ be a homeomorphism of $E^{k}$ onto itself so that $f(L)$ is locally polyhedral at each point of $f(L)-f(p)$. We use Lemma 2 of [2] to obtain a homeomorphism $g$ of $E^{k}$ onto itself such that $g f(L)$ is polyhedral. We then use Theorem 5 of [6] to obtain a homeomorphism $h$ of $E^{k}$ onto itself that carries $g f(L)$ onto $B_{1}$.

For each positive integer $n$ we may establish the following theorem.

Theorem $1_{n}$. Let $F_{n}=\bigcup_{i=1}^{n} A_{i}$ be an $n$-frame, with distinguished point $p$, such that $A_{i}, i=1,2, \cdots, n$, is locally flat at each point of $A_{i}-p$. Then $F_{n}$ is tame.

Proof. Theorem $1_{1}$ has been proved in Lemma 2 . We next assume that Theorem $1_{n-1}, n \geqq 2$, is true and proceed to show that Theorem $1_{n}$ is true.

Let $F_{n}=\bigcup_{i=1}^{n} A_{i}$ be an $n$-frame which satisfies the hypotheses of Theorem $1_{n}$. There is a homeomorphism $\phi_{1}$ of $E^{k}$ onto itself such that $F_{n-1}=F_{n}-\left(A_{n}-p\right)$ is carried onto $G_{n-1}$. Since $\phi_{1}\left(A_{n}\right)$ is tame, there is a neighborhood $V$ of $\phi_{1}\left(A_{n}-p\right)$ which does not intersect $G_{n-1}$, and, by Lemma 1 , a homeomorphism $\phi_{2}$ on $E^{k}$ such that $\phi_{2} \phi_{1}\left(A_{n}\right)$ is locally polyhedral at each point of $\phi_{2} \phi_{1}\left(A_{n}-p\right)$ and $\phi_{2}$ is fixed outside $V$. We next construct a homeomorphism $\phi_{3}$ on $E^{k}$ such that $\phi_{3} \phi_{2} \phi_{1}\left(A_{n}\right)=B_{n}$ and $\phi_{3}$ is fixed on $G_{n-1}$. The homeomorphism $\phi_{3} \phi_{2} \phi_{1}$ will then carry $F_{n}$ onto $G_{n}$ and the proof of Theorem $1_{n}$ will be complete. Since the construction of $\phi_{3}$ is almost identical with that used in the proof of Lemma 2 of [2], we will only give an outline of the construction.

We use the local connectivity of $\phi_{2} \phi_{1}\left(A_{n}\right)$ to find a sequence $\left\{V_{m}\right\}_{m=1}^{\infty}$ of closed cubical neighborhoods of the origin such that (1) the end points of $B_{n}$ and $\phi_{2} \phi_{1}\left(A_{n}\right)$, different from the origin, are outside $V_{1},(2)$ the diameters of the $V_{m}$ converge to zero, and (3) if $L$ is any subarc of $\phi_{2} \phi_{1}\left(A_{n}\right)$ whose end points lie in $V_{m}$, then $L$ is contained in Int $V_{m-1}$. We will further assume that $\phi_{2} \phi_{1}\left(A_{n}\right) \cap \mathrm{Bd} V_{2 m}$ is a finite set of points and that no pair of components of $\phi_{2} \phi_{1}\left(A_{n}\right)$ $-V_{2 m}$ share a common end point.

For each positive integer $m$ let $u_{m 1}, \cdots, u_{m k(m)}$ be the components of $\phi_{2} \phi_{1}\left(A_{n}\right)$-Int $V_{2 m}$ which have both end points on Bd $V_{2 m}$. Since there can be no knotting or linking of polyhedral simple closed curves 
in an euclidean space of dimension greater than three, each of the $u_{m i}$ may be moved into Int $V_{2 m}$ without moving points outside $V_{2 m-1}-V_{2 m+1}$ or on $G_{n-1}$. Thus we may construct a semilinear homeomorphism $f_{m}$ such that $f_{m} \phi_{2} \phi_{1}\left(A_{n}\right) \cap \mathrm{Bd} V_{2 m}$ is a single point, and $f_{m}$ is the identity on $E^{k}-\left(V_{2 m-1}-V_{2 m+1}\right)$ and on $G_{n-1}$. A homeomorphism $f$ is defined by $f(x)=x$, if $x \in E^{k}-V_{1}, f(x)=f_{m}(x)$, if $x \in V_{2 m-1}-V_{2 m+1}$, $m=1,2, \cdots$, and $f$ carries the origin onto itself.

Let 0 denote the origin, let $x_{0}$ be the end point of $B_{n}$ different from $0, x_{m}=B_{n} \cap \mathrm{Bd} V_{2 m}, m=1,2, \cdots, y_{0}$ the end point of $f \phi_{2} \phi_{1}\left(A_{n}\right)$ different from 0 , and $y_{m}=f \phi_{2} \phi_{1}\left(A_{n}\right) \cap \mathrm{Bd} V_{2 m}, m=1,2, \cdots$. Let $g_{0}$ be a semilinear homeomorphism on $E^{k}$ such that $g_{0}$ is fixed on $V_{1}$ and $g_{0}\left(y_{0}\right)=x_{0}$. For $m=1,2, \cdots$, let $g_{m}$ be a semilinear homeomorphism on $E^{k}$ such that $g_{m}$ is fixed outside $V_{2 m-1}-V_{2 m+1}$ and on $G_{n-1}$, and $g_{m}\left(y_{m}\right)=x_{m}$. A homeomorphism $g$ is then defined by $g(x)=g_{0}(x)$, for $x \in E^{k}-V_{1}, g(x)=g_{m}(x)$, for $x \in V_{2 m-1}-V_{2 m+1}$, and $g(0)=0$.

Again since there can be no knotting or linking of polyhedral simple closed curves in $E^{k}$, we may construct homeomorphisms $h_{m}$ with the following properties. The map $h_{0}$ is fixed on $V_{2}$ and on $G_{n-1}$, and carries the subarc of $g f \phi_{2} \phi_{1}\left(A_{n}\right)$ from $x_{0}$ to $x_{1}$ onto the linear segment $\left[x_{0} x_{1}\right]$. For $m=1,2, \cdots, h_{m}$ is fixed on $E^{k}-\left(V_{2 m}-V_{2 m+2}\right)$ and on $G_{n-1}$, and carries the subarc of $g f \phi_{2} \phi_{1}\left(A_{n}\right)$ from $x_{m}$ to $x_{m+1}$ onto the linear segment $\left[x_{m} x_{m+1}\right]$. We set $h(x)=h_{0}(x)$, if $x \in E^{k}-V_{2}, h(x)=h_{m}(x)$, if $x \in V_{2 m}-V_{2 m+2}$, and $h(0)=0$. Finally we take $\phi_{3}=h g f$.

COROllary $1_{n}$. There are no mildly wild $n$-frames in $E^{k}$.

Proof. Suppose $F_{n}=\bigcup_{i=1}^{n} A_{i}, n>1$, is an $n$-frame such that for each $j=1,2, \cdots, n, F_{n}-\left(A_{j}-p\right)$ is tame. Then each $A_{j}$ is tame and, by Theorem $1_{n}, F_{n}$ is tame.

Corollary $2_{2}$. If $A_{1}$ and $A_{2}$ are tame arcs in $E^{k}$, meeting only in a common end point, then $A_{1} \cup A_{2}$ is tame.

Theorem 2. If $A$ is a wild simple closed curve (arc) in $E^{k}$ and $E$ is the set of points at which $A$ fails to be locally flat, then $E$ contains a Cantor set.

Proof. For $A$ a simple closed curve, we know that $E$ is nonempty [5]. By the definition of local flatness, the set of points at which $A$ is locally flat is an open subset of $A$, and $E$ is therefore closed. If we establish that $E$ has no isolated points, there are two possibilities. First, $E$ may be totally disconnected, in which case $E$ is a Cantor set. Secondly, $E$ may have a nondegenerate component $K$, in which case $K$ is either an arc or $K=A$. 
In order to show that there are no isolated points of $E$, let us consider a point $q$ of $A$ such that there is a neighborhood $U$ of $q$, relative to $A$, with $A$ locally flat at each point of $U-q$. We select two arcs $A_{1}$ and $A_{2}$ of $A$ such that $A_{1} \cup A_{2} \subset U$ and $A_{1} \cap A_{2}=q$. By Lemma 2 and Corollary $2_{2}, A_{1} \cup A_{2}$ is tame, and $A$ is therefore locally flat at $q$.

This proves Theorem 2 for $A$ a simple closed curve. A similar argument establishes the theorem for $A$ an arc.

3. Added in proof. Suppose that $M$ is a finite 1-complex topologically embedded in $E^{k}, k>3, V$ is the set of vertices of $M$, and $M$ is locally flat at each point of $M-V$ (equivalently, each 1-simplex of $K$ is locally flat at each of its interior points). By first applying Homma's Theorem, as in Lemma 1, we may construct a homeomorphism $f$ of $E^{k}$ onto itself such that $f(M)$ is locally polyhedral at each point of $f(M)-f(V)$. Then, by applying the technique of proof used in Theorem $1_{n}$ at each point of $f(V)$, we may construct a homeomorphism $g$ of $E^{k}$ onto itself such that $g f(M)$ is polyhedral. Thus we see that $M$ is tame. An immediate corollary is that a finite 1-complex, topologically embedded in $E^{k}, k>3$, is tame if and only if each simplex is tame.

\section{REFERENCES}

1. W. A. Blankinship, Generalization of a construction of Antoine, Ann. of Math. (2) 53 (1951), 276-297.

2. J. C. Cantrell and C. H. Edwards, Jr., Almost locally polyhedral curves in euclidean n-space, Trans. Amer. Math. Soc. 107 (1963), 451-457.

3. $\mathrm{H}$. DeBrunner and R. Fox, $A$ mildly wild imbedding of an $n$-frame, Duke Math. J. 27 (1960), 425-429.

4. P. H. Doyle, Certain manifolds with boundary which are products, Mimeographed notes, Virginia Polytechnic Institute, Blacksburg, Va.

5. H. Gluck, Unknotting $S^{1}$ in $S^{4}$, Bull. Amer. Math. Soc. 69 (1963), 91-94.

6. V. K. A. M. Gugenheim, Piecewise linear isotopy and embedding of elements and spheres. I, Proc. London Math. Soc. 3 (1953), 29-53.

UNIVERSITY OF GEORGIA 